\title{
Hypoxia alters P-gp Expression and Activity in Three Different Rat Intestinal Modles: Implications for Levofloxacin Delivery
}

Research Article

Li Wen-bin ${ }^{1 \&}$, Jiang Ze-juan ${ }^{2 \&}$, Wang Rong ${ }^{2 \&}$, Zhao An-peng ${ }^{2}$, Li Xue ${ }^{2}$, Luo Bing-Feng ${ }^{2}$, Yang Xi ${ }^{3 *}$

${ }^{1}$ Department of Pharmacy, The 940th Hospital of Joint Logistics Support Force of Chinese People's Liberation Army, Lanzhou, China. ${ }^{2}$ Department of Pneumolog, The 940th Hospital of Joint Logistics Support Force of Chinese People's Liberation Army, Lanzhou, China. ${ }^{3}$ College of Science, Gansu Agricultural University, Lanzhou, China.

\& These authors contributed equally to this work and should be considered co-first authors.

\section{Abstract}

Objective: To investigate the difference in P-glycoprotein (P-gP) expression and the absorption of levofloxacin in rat small intestines between high altitude and plain areas.

Methods: Wistar rats kept in Shanghai (representing the plain area) were used as the normoxic group, and rats rapidly exposed to high altitude via flights were used as the hypoxic group. Reverse transcription-quantitative PCR and western blotting were performed to assess the mRNA and protein expression of P-gp, respectively. The everted intestinal sac model, the in-situ single-pass perfused intestinal model, and the whole animal model were used to investigate levofloxacin absorption in rat intestines.

Results: The mRNA and protein expression levels of P-gp were significantly decreased (by $50.80 \%$ and $71.30 \%$ ) in the hypoxic group compared with those in the normoxic group. In the everted intestinal sac model, the maximum values of levofloxacin absorption in the hypoxic group were $19.99 \%, 29.29 \%$, and $45.47 \%$ in the duodenum, jejunum, and ileum segments, respectively, at $75 \mathrm{~min}(\mathrm{P}<0.05)$. The permeability parameters (Peff) of the hypoxic group increased by $3256.16 \%, 226.00 \%$, $77.74 \%$, and $141.00 \%$ at $30-60 \mathrm{~min}, 60-90 \mathrm{~min}, 90-120 \mathrm{~min}$, and $120-150 \mathrm{~min}$, respectively $(\mathrm{P}<0.05)$. In the hypoxic group, the area-under-the-curve of plasma P-gp increased by 5.05-, 4.90-, and 3.85-fold, respectively, after the oral administration of low-, medium-, and high-dose levofloxacin compared with those in the normoxic group, while the peak plasma concentration increased by 6.04-, 3.28-, and 2.87-fold, respectively.

Discussion: Hypoxia downregulated P-gp expression and increased levofloxacin absorption in rats at high altitudes. Understanding differential mechanisms of drug transporters under hypoxia is important for informed drug administration at high altitudes.

\section{Introduction}

Increasing scientific evidence indicates that hypoxia may affect the pharmacokinetics of drugs at high altitudes. Studies have focused on variations in drug-metabolizing enzyme activities and protein expression under hypoxia, which are critical factors in mediating drug metabolism $[6,9,10,12,18]$. However, few studies have addressed the effect of drug transporters on Pharmacokinetics [7]. Drug transporters, especially P-glycoprotein (P-gp), are important molecules that not only contribute to drug metabolism but also other processes such as absorption, distribution, metabolism, and excretion (ADME) in vivo. P-gp is an important member of the $\mathrm{ABC}$ drug transporter family (140-180 kDa), mainly expressed in the small intestine, hepatocytes, kidney proximal tubules, and the blood-brain barrier. P-gp plays a key role in mediating drug export from cells, protects the body against foreign substances, and contributes to ADME in terms of intestinal absorption [3], biliary excretion, and urinary excretion [14, 19]. Some studies have indicated that drug transporters may play a key role in pharmacokinetic changes at high altitudes. Several reports have investigated how hypoxia could affect the mRNA and protein expression of P-gp under hypoxic tumor microenvironment and

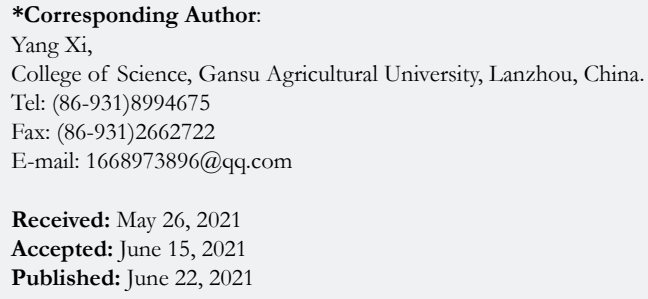


chemical hypoxia [4]. achieved intermittent hypoxic exposure (at 4-min intervals) by housing rats in a plexiglass chamber for $12 \mathrm{~h}$ each day for 14 consecutive days [4]; while protein expression was not significantly different, the mRNA expression of Abcb1a was significantly upregulated in the liver. In contrast $[6,7]$. demonstrated that the protein levels of P-gp were significantly increased by $77 \%$ in the livers of hypoxic rats, but the mRNA expression of P-gp was not affected when the rats were exposed to an FiO2 of $8 \%$ in a plexiglass chamber $(0.75 \mathrm{~m} \times 1.20 \mathrm{~m} \times 1.25 \mathrm{~m})$ to induce acute moderate hypoxia for $48 \mathrm{~h}[3,7]$. reported significant upregulation of P-gp mRNA and protein in human colon carcinoma tissues and four cell lines (HCT-116, HT-29, LoVo, and SW480) in the hypoxic groups $[3,16]$. demonstrated that P-gp was upregulated in hepatoma tumor spheroids subjected to chemical hypoxia induced by either cobalt chloride or desferrioxamine $[16,11]$. reported that P-gp expression was significantly increased during the tumorigenesis of colorectal cancer; the expression was generally lower in poorly differentiated tumors and higher in well-differentiated tumors[1,8,11,17]. However, few reports have investigated how P-gp affects the pharmacokinetics of its substrate under hypoxic conditions at high altitudes. Levofloxacin, a specific substrate of P-gp[15], is a fluoroquinolone antibiotic used for treating different types of bacterial infections at high altitudes. It is a synthetic broad-spectrum antibacterial agent that acts in a concentration-dependent manner and whose effective bactericidal activity depends on the ratio of maximum concentration $\left(\mathrm{C}_{\max }\right)$ to minimum inhibitory concentration. Intestinal absorption is a critical factor affecting the concentration of levofloxacin in the plasma, and the absorption of levofloxacin depends on P-gp expression that acts as an efflux pump and is responsible for decreased drug accumulation in cells. The present study investigated differential expression of P-gp between high altitude and plain areas, and examined the changes in levofloxacin absorption in the rat intestine using an everted rat intestinal sac model, an in-situ single-pass perfused rat intestinal model, and a whole rat model.

\section{Material And Methods}

\section{Materials}

Healthy male wistar rats were purchased from ShangHai SLAC Laboratory Animal co. LTD (certification number was 2007000524909). ViiA TM 7 DX Real-Time QPCR System (Applied Biosystems, Inc., Foster City, CA, USA), ChemiDoc ${ }^{\text {TM }}$ XRS Imaging System (Bio-Rad Laboratories, California, USA), HPLC grade formic acid was purchased from DIMA Technology Inc. (Richmond, USA). The levofloxacin $2 \mathrm{~mL} 0.2 \mathrm{~g}$ was purchased from HuNan Wuzhoutong pharmaceutical co. LTD (China). Sodium pentobarbital $(10 \mathrm{mg} \cdot \mathrm{mL}-1)$ was purchased from Shanghai longsheng chemical co. LTD (China). ProteoExtract ${ }^{\circledR}$ Transmembrane Protein Extraction Kit( (Novagen, EMD Chemicals Inc., Darmstadt, Germany), The rabbit anti-P-gp antibody and anti$\beta$-actin antibody were purchased from Abcam (Cambridge UK, Art.No: ab170904, ab8227). Secondary antibodies were obtained from ZSGB-BIO (Beijing, China). HPLC grade acetonitrile was purchased from Merck Drugs \& Biotechnology (EMD Chemicals Inc., Darmstadt, Germany). All reagents used for quantitative real-time polymerase chain reaction (PCR) were purchased from Takara Biotechnology Inc. (Shiga, Japan). All other chemicals were of the highest quality available from commercial sources. All animal protocols were consistent with the standards for the
Care and Use of Laboratory Animals Guidelines at the Lanzhou General Hospital of Lanzhou Military Command.

\section{Animals and Experimental preparation}

Wistar rats were obtained from the SLAC laboratory animal CO.LTD, Shanghai, China, and bred in the animal laboratory of Second medical university for a week before experiment. During the experimental period, the animals were remained normal water and food. 12 rats were randomly divided into normoxic group, (Shanghai, $31^{\circ} 22^{\prime} \mathrm{NW}, 121^{\circ} 48^{\prime} \mathrm{EL}, 55$ meters, $20-25^{\circ} \mathrm{C}$ Atmospheric pressure: $95.60 \mathrm{Kpa}$ ), which allowed free access to regular rodent diet and water, hypoxia group group (Maduo, 34 $92^{\prime} \mathrm{NW}$, $98^{\circ} 26^{\prime} \mathrm{EL}, 4300$ meters, $20-25^{\circ} \mathrm{C}$, Atmospheric pressure 59.10 Kpa ), which rapidly into high altitude by flight and exposed for 3 days after normally fed at Maduo.

\section{mRNA and Protein expression analysis of P-gp}

Whole small intestine was collected from unprocessed rats at high altitude and plain area respectively. The Quantitative real-time PCR analysis was performed based on the manufacturer's standard protocols. The following oligonucleotide primers sequences were used for amplification of rat P-gp (Abcb1a): forward 5'CCT GAA ATC CAG CGG CAGA -3' and reverse 5'- ATG TAT CGG AGT CGC TTG GTG AG-3', $\beta$-actin: forward 5'- GGA GAT TAC TGC CCT GGC TCC TA-3'and reverse 5'- GAC TCA TCG TAC TCC TGC TTG CTG-3', The first step of PCR is initial denaturation at $95^{\circ} \mathrm{C}$ for $30 \mathrm{~s}$. Secondly, performed a amplification reaction $\left(95^{\circ} \mathrm{C}\right.$ for $5 \mathrm{~s}$ and $60^{\circ} \mathrm{C}$ for $\left.34 \mathrm{~s}\right)$ by 40 cycles. Thirdly, generated melting curve profile $\left(95^{\circ} \mathrm{C}\right.$ for $15 \mathrm{~s}, 60^{\circ} \mathrm{C}$ for $1 \mathrm{~min}, 95^{\circ} \mathrm{C}$ for $15 \mathrm{~s}$ ) for the high purity of amplification substances. Finally, determine the specificity of PCR products by agarose gel electrophoresis. Each sample was assayed in triplicate. The expression of the entire target gene was normalized to $\beta$-actin as reference gene and calculated from the cycle threshold (CT) value of each sample using the ViiA ${ }^{\text {TM }} 7$ Software version 1.1. The fold changes in P-gp expression between two groups were calculated by the $2^{-\Delta \mathrm{CT}}$ equation method. The intestinal membrane fraction prepared in accordance with the instructions of ProteoExtract ${ }^{\circledR}$ Transmembrane Protein Extraction Kit. Proteins from tissue homogenates (20ug) were separated on polyacrylamide gel (SDS-PAGE) by electrophoresis. The following antibodies were used: P-gp (Abcam, UK, Art.No, ab170904, 1:5000), and $\beta$-actin (Abcam, UK, Art.No, ab8227, 1:1000). P-gp protein levels were normalized to $\beta$-actin levels.

\section{Everted intestinal sacs model (For assessing absorptivity of levofloxacin)}

Rats were fasted overnight before the experiment, anesthetized by injecting sodium pentobarbital $(10 \mathrm{mg} \bullet \mathrm{mL}-1) 0.4 \mathrm{ml}$ per $100 \mathrm{~g}$ in enterocoelia. Rats were dissected for collecting the small intestine, carefully peel off the mesentery, relevant fat and blood vessels immediately, rinsing small intestine by cold Tyrode's solution. Scissoring $10 \mathrm{~cm}$ duodenum, jejunum and ileum in natural state do not stretch the intestine carefully flip the end of the intestine with a glass rod to make the small intestine mucosa facing outward, and clean in the Tyrode solution, with mousse wire ligation anal end, an 8-g stainless steel weight was attached to the ligated end of the sac to maintain it in a vertical position during the experiment. The other end is fixed to a PE diameter about $0.3 \mathrm{~mm}$ for sample port. 
A Krobs-Ringer test solution of the same volume $(1 \mathrm{~mL})$ was injected into the intestine from the sampling port using a syringe and placed vertically in a test tube which containing drug solution, the drug solution was made in Tyrode solution (levofloxacin: 0.5 $\left.\mathrm{mg} \cdot \mathrm{mL}^{-1}\right)$, make sure the intestinal fluid level higher than the test tube. There are two groups in high altitude, no oxygen and $50 \% \mathrm{O}_{2}$ were respectively introduced into the test tube as hypoxia groups, and $95 \% \mathrm{O}_{2}$ was introduced into the test tube at plain area as the normal group, and the whole device was put into a constant temperature water bath at $37^{\circ} \mathrm{C}$, extract $30 \mathrm{uL}$ sacs fluid from the sample port for determination each time.

Situ single-pass perfused rat intestinal model (For calculating the permeability parameters of levofloxacin)

Rats were fasted overnight before the experiment, anesthetize rats with ip injection of sodium pentobarbital $\left(10 \mathrm{mg}^{\bullet} \mathrm{mL}^{-1}\right) 0.4 \mathrm{ml}$ per $100 \mathrm{~g}$, open the abdominal cavity by a middle incision of 3-4 $\mathrm{cm}$ and locate the jejunum, cannulate a segment of the jejunum (about 10-15 cm) using PE350 tubing (precut to length), and connected to the perfusate pipe. The tube are secured with a surgical silk suture, place a portion of the intestine within the abdominal cavity after the cannulation, lay the rest of the intestinal segment flat on the abdominal surface, the whole area is then covered by a paper towel wetted with normal saline, a piece of plastic wrap is put on the towel to keep the intestinal segments moist. Set the muti-channel infuse pump to a desired flow rate $\left(0.2 \mathrm{ml} \cdot \mathrm{min}^{-1}\right)$, one channel connected to the perfusate pipe, another to the control fluid pipe, Keep the circulating water bath at $37^{\circ}$ to maintain the temperature of the perfusate constant clear the intestine for 30 min by using the perfusate which containing the compound of levofloxacin and Phenolsulfonphthalein (non absorbable water flux marker) in Krobs-Ringer buffer, then collect the perfusate at every 30 min intervals afterward until all four samples are collected at $120 \mathrm{~min}$. Measure the length of perfused intestine by wetting them with normal saline $\left(4^{\circ} \mathrm{C}\right)$ and carefully laying them flat without stretching, prepare the collected perfusate for further measurements. This method measures the steady-state uptake of a compound from the perfusate by determining the rate of disappearance from the perfusate and uses the rate of disappearance to calculate an unbiased intestinal wall permeability $\left(\mathrm{P}_{\text {eff }}\right)$, the Peff of a compound is calculated using the following equations:

$P_{e f f}=\left(1-C_{m} / C_{0}\right) / 4 G_{\mathrm{z}} ; P_{a q}=\left(A\left(G_{z}\right)^{1 / 3}\right)^{-1} ; \mathrm{A}=10.0 G_{\mathrm{z}}+1.01$

$0.004 \leq G_{z} \leq 0.01$

$\mathrm{A}=4.5 G_{\mathrm{z}}+1.0650 .01 \leq G_{\mathrm{z}} \leq 0.03 ; \mathrm{z} \mathrm{A}=2.5 G_{\mathrm{z}}+1.1250 .03 \leq G_{\mathrm{z}}$.

$\mathrm{C}_{0}$ and $\mathrm{C}_{\mathrm{m}}$ are inlet and outlet concentrations, respectively; $\mathrm{Gz}$ is a scaling factor that incorporates flow rate $(\mathrm{Q})$, intestinal length $(\mathrm{L})$, and diffusion coefficients (D) to make the permeability dimensionless; and $\mathrm{A}$ is a correction factor for the aqueous resistance of the intestine. $\mathrm{Cm}$ was adjusted for water flux, as indicated by the concentration of Phenolsulfonphthalein, a non absorbable marker compound. For experiments performed in the small intestine, the data points are discarded if the water flux exceeds $0.55 \% / \mathrm{cm}$.

Whole animal model (For evaluate pharmacokinetics of levofloxacin)

Rats were fasted overnight before the experiment, and orally administered three single dose of levofloxacin respectively (10 $\mathrm{mg} \bullet \mathrm{kg}^{-1}, 20 \mathrm{mg} \bullet \mathrm{kg}^{-1}$ and $\left.40 \mathrm{mg} \bullet \mathrm{kg}^{-1}\right)$ both in high altitude and plain area, blood samples $(0.25 \mathrm{ml})$ were collected from retinal venous plexus at $0 \mathrm{~min}, 20 \mathrm{~min}, 40 \mathrm{~min}, 1 \mathrm{~h}, 1.5 \mathrm{~h}, 2 \mathrm{~h}, 3 \mathrm{~h}, 4 \mathrm{~h}, 6$ $\mathrm{h}, 8 \mathrm{~h}, 12 \mathrm{~h}$ and $24 \mathrm{~h}$ after administration.

\section{Concentration determination of levofloxacin}

Shimadzu LC-20AD series HPLC system (Shimadzu, Japan) coupled with Applied Biosystems Sciex Q-trapTM triple quadrupoles tandom mass spectrometer (AB, USA) via an electrospray ionization (ESI) source were used for analysis. Samples were separated on Shim-pack XR-ODS column $(3.0 \mathrm{~mm} \times 75 \mathrm{~mm}, 2.0 \mu \mathrm{m}$, Agilent Technologies, USA) maintained at $20^{\circ} \mathrm{C}$. The mobile phase consisted of Acetonitrile-Water - Formic acid (55:45:0.1, v/v/v), and was delivered at a flow rate of $0.40 \mathrm{~mL} \cdot \mathrm{min}^{-1}$. LC-MS-MS was equipped with an electrospray ion source operating at 300 ${ }^{\circ} \mathrm{C}, 5.5 \mathrm{kV}$. The declustering potential and collsion energy was set at $32 \mathrm{~V}$ and 25 psi. The analysis was carried out using multiple reaction monitoring (MRM) for the following transitions: Levofloxacin $(\mathrm{m} / \mathrm{z} 362.2 \rightarrow 318.2)$. The measured intensities of the two Levofloxacin transitions were summed to achieve the necessary sensitivity.

\section{Statistical analysis}

The data were expressed as mean \pm S.D and were analyzed by the Student's t-test to do comparison between each two groups. Use One-way analysis of variance (ANOVA) to do comparison between three or more groups. $\mathrm{p}<0.05$ was considered to be statistically significant.

\section{Results}

mRNA and Protein expression changes of P-gp after acute exposed to high altitude

The results of quantitative real-time PCR are summarized in FIGURE 1A. The figure shows that the P-gp mRNA level was significantly decreased $50.80 \%$ in hypoxic group compared with normoxic group. Specific bands were detected at around $170 \mathrm{kDa}$ for P-gp (FIGURE 1B), the protein relative expression levels of P-gp declined by $71.30 \%$ in hypoxic group compared with normoxic group (FIGURE 1B).

\section{Absorptivity of levofloxacin in Everted intestinal sacs model}

As shown in FIGURE 2A, at the time of 15 minutes, there have no significant difference in the absorption of levofloxacin in duodenum segment, the levofloxacin absorption increased by $3.18 \%$ and $5.09 \%$ respectively in jejunum and ileum segments in no oxygen group compared with the $95 \% \mathrm{O}_{2}$ group $(\mathrm{P}<0.05)$. The levofloxacin absorption of $50 \% \mathrm{O}_{2}$ group increased by $3.41 \%$ in ileum segment compared with the $95 \% \mathrm{O}_{2}$ group $(\mathrm{P}<0.05)$. As shown in FIGURE 2B, at the time of 45 minutes, the levofloxacin absorption of no oxygen group increased by $19.99 \%, 29.29 \%$ and $38.83 \%$ respectively in duodenum, jejunum and ileum segments compared with the $95 \% \mathrm{O}_{2}$ group $(\mathrm{P}<0.05)$. The no oxygen group absorption increased by $10.92 \%$ in duodenum compared with the $50 \% \mathrm{O}_{2}$ group $(\mathrm{P}<0.05)$. The levofloxacin absorption of $50 \% \mathrm{O}_{2}$ group increased by $8.18 \%, 18.27 \%$ and $32.34 \%$ re- 
spectively in duodenum, jejunum and ileum segments compared with the $95 \% \mathrm{O}_{2}$ group $(\mathrm{P}<0.05)$. As shown in FIGURE 2C, at the time of 75 minutes, the levofloxacin absorption of no oxygen group increased by $18.19 \%, 37.75 \%$ and $45.47 \%$ respectively in duodenum, jejunum and ileum segments compared with the $95 \%$ $\mathrm{O}_{2}$ group $(\mathrm{P}<0.05)$. The no oxygen group absorption increased by $22.90 \%$ in duodenum compared with the $50 \% \mathrm{O}_{2}$ group $(\mathrm{P}<0.05)$. The levofloxacin absorption of $50 \% \mathrm{O}_{2}$ group increased by $9.86 \%, 27.65 \%$ and $37.83 \%$ respectively in duodenum, jejunum and ileum segments compared with the $95 \% \mathrm{O}_{2}$ group $(\mathrm{P}<0.05)$. As shown in FIGURE 3, the slope of the absorption curve was steeper when oxygen was insufficient.

\section{$\mathbf{P}_{\text {eff }}$ of levofloxacin in Situ single-pass perfused rat intestinal model}

Rat characteristics: The lengths of small intestine between normoxic and hypoxic groups were $(66.67 \pm 1.75) \mathrm{cm}$ and $(66.33 \pm 2.25)$ $\mathrm{cm}$ respectively, and there was no significant difference between the two groups, $\mathrm{P}>0.05$.

Check perfusion fluid volume change: Through calibration, the volume changes of perfusate in different perfusion period were $5.33 \%, 2.50 \%,-1.83 \%$, and $3.50 \%$ in normoxic group, the volume changes in the hypoxic group were $-1.17 \%,-0.66 \%$, $6.17 \%,-2.16 \%$. There was no significant difference in the volume

Figure 1. Mdr1 is influenced by hypoxia on the mRNA and protein level in small intestine.

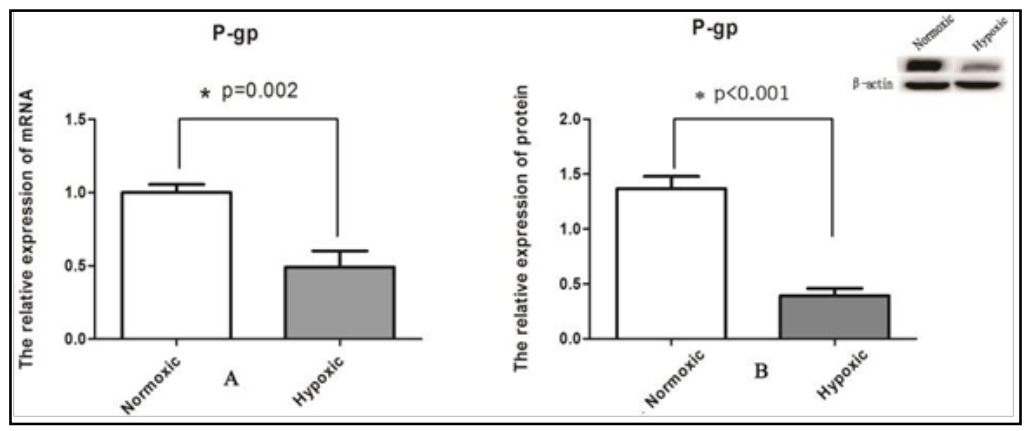

mRNA expression was detected by qRT-PCR (A) and protein expression was detected by western blot (B). Hypoxic group rats were exposed to the high altitude for 3 days. Each data was demonstrated as mean \pm S.D. $(n=6)$.

Figure 2. The absorption of levofloxacin is enhanced by hypoxia at the 15(A), 45(B) and 75(C) min in the everted intestinal sacs model.

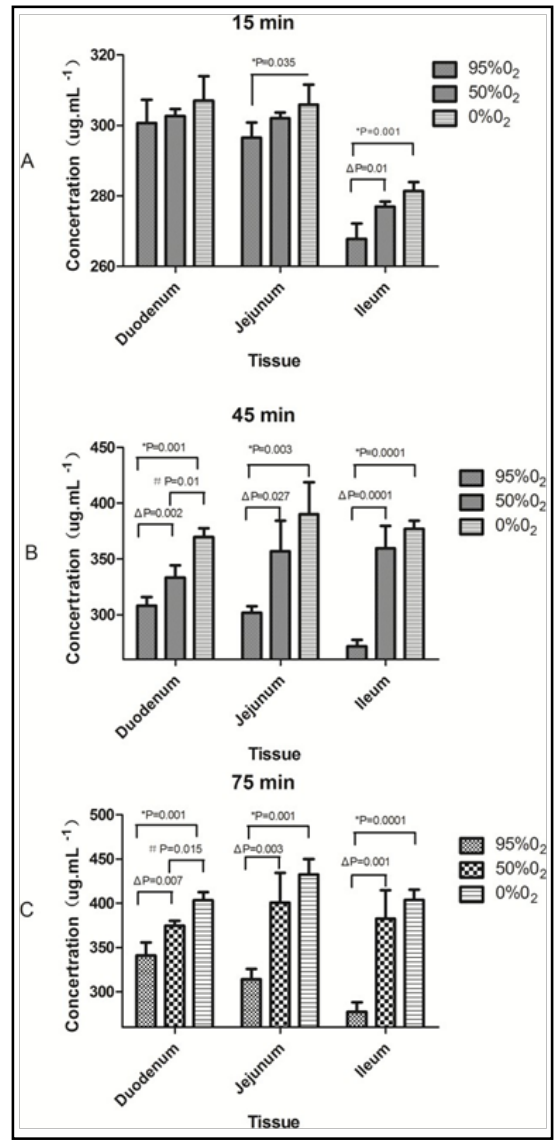

Hypoxic group rats were exposed to the high altitude for 3 days. Each data was demonstrated as mean \pm S.D. ( $=6$ ). Duodenum, jejunum and ileum segments' length are $10 \mathrm{~cm}$. 
Figure 3. The increased tendency of levofloxacin absorption in duodenum(A), jejunum (B), and ileum (C) segments as time prolonged.

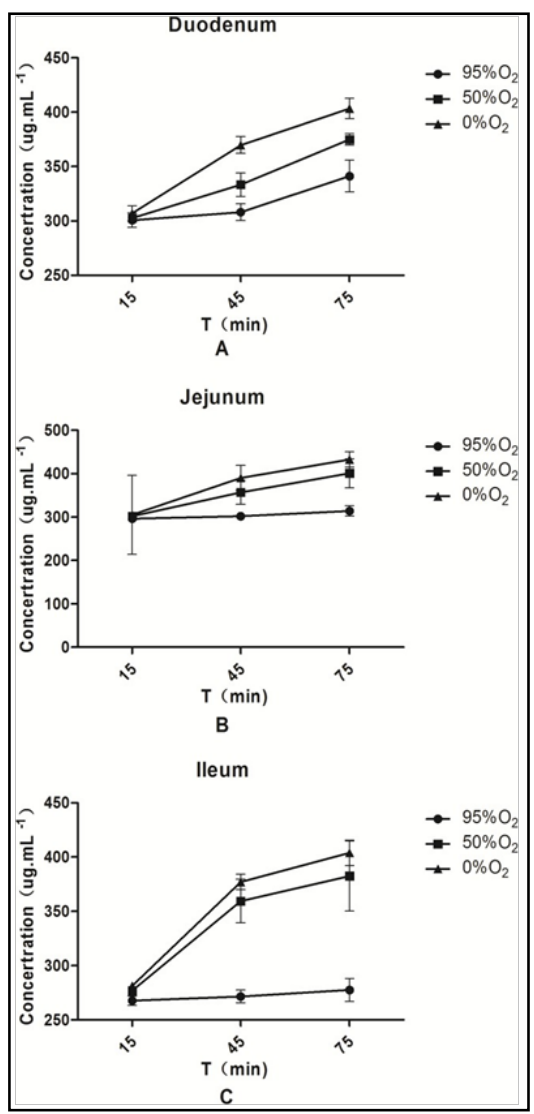

Hypoxic group rats were exposed to the high altitude for 3 days. Each data was demonstrated as mean \pm S.D. $(n=6)$.

change between the two groups, $\mathrm{P}>0.05$ (See TABLE 1$)$. The rate of change of volume is within the allowable range, indicating successful modeling.

Levofloxacin Concentration in Perfusate: The concentration of levofloxacin was measured and adjusted as shown in TABLE 2. The results showed that there have a significant difference in drug absorption between hypoxic group and normoxic group at each time point, and the drug absorption was significantly accelerated after hypoxia, the concentration of perfusate in the hypoxia group decreased by $32.83 \%, 59.20 \%, 28.18 \%$, and $59.63 \%$ respectively in 30-60 $\mathrm{min}, 60-90 \mathrm{~min}, 90-120 \mathrm{~min}$, and $120-150$ $\min (\mathrm{P}<0.05)$.

The permeability parameters of levofloxacin: As shown in FIGURE 4, the permeability parameters of levofloxacin $\left(\mathrm{P}_{\text {eff }}\right)$ in the hypoxic group increased by $56.16 \%, 226.00 \%, 77.74 \%$, and $141.00 \%$ respectively at $27130-60 \mathrm{~min}, 60-90 \mathrm{~min}, 90-120 \mathrm{~min}$, and $120-150 \mathrm{~min}(\mathrm{P}<0.05)$.

\section{Pharmacokinetics of levofloxacin in whole animal model}

The mean plasma concentration-time profiles of levofloxacin are shown in FIGURE 5, we can found statistical significance between the two groups. AUC, Cmax representing the degree of absorption after oral administrated levofloxacin, AUC had seriously increased by 5.05, 4.90 and 3.85 folds severally after oral administrated low, middle and high dose levofloxacin in hypoxic group compared with normoxic group, while $\mathrm{C}_{\max }$ had seriously increased by $6.04,3.28$ and 2.87 folds. Other pharmacokinetic parameters are summarized in TABLE 3.

\section{Discussion}

Both micro-anoxic tumor environment and chemical hypoxia have been shown to upregulate P-gp expression; however, it was unclear whether P-gp expression would also be upregulated under hypoxia at high altitudes. Contrary to the earlier findings, the outcomes of the present study indicate that both the mRNA and protein expression of P-gp were downregulated in the small intestine. This may be attributed to different types of tissues used between studies, as normal intestinal tissues were used in the present study, while previous studies used tumor tissues. The hypoxic conditions also differed, as the present study investigated hypoxia at high altitudes, while previous studies used the micro-anoxic tumor environment or chemical hypoxia.

P-gp is mainly expressed on the brush border membrane in intestinal epithelial cells to transports drugs from intestinal epithelial cells into the enteric canal. The decreased expression of P-gp could lead to decreased drug absorption as observed in the present study, could reduce the efflux of its substrate, and enhance its absorption. To verify this hypothesis, levofloxacin was selected as the specific substrate of P-gp for testing in three different absorption models, and results from the three different models confirmed that levofloxacin absorption increased in the small intestine after exposure to acute hypoxia at high altitudes. Firstly, the absorption of levofloxacin was investigated in different segments of the intestine-the duodenum, jejunum, and ileum-using the everted intestinal sac model. Hypoxia significantly increased the absorption of levofloxacin in all three segments of the intestine, which corresponded to the downregulated expression of P-gp. 
Table 1 Check perfusion fluid volume change with Phenolsulfonphthalein $(X \pm S, n=6)$.

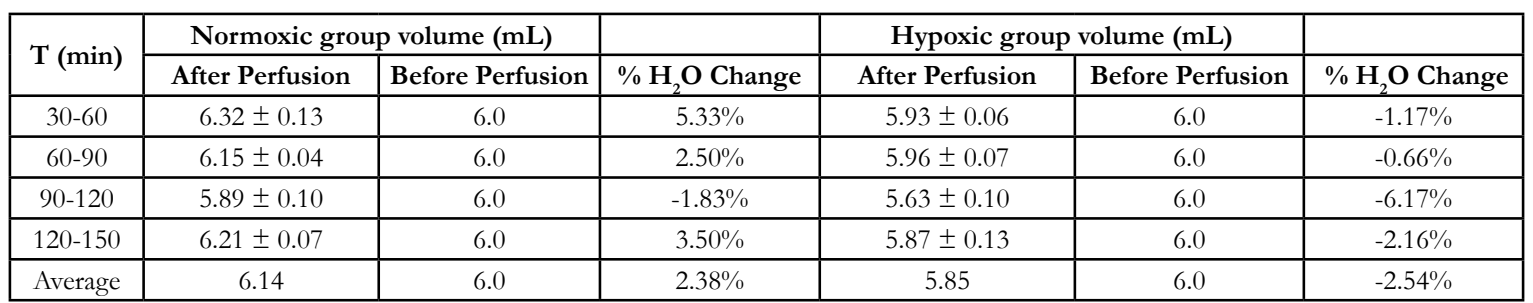

Table 2. Levofloxacin Concentration in Perfusate $(X \pm S, n=6)$.

\begin{tabular}{|c|c|c|c|c|}
\hline \multirow{2}{*}{ T (min) } & \multicolumn{2}{|c|}{$\begin{array}{c}\text { Normoxic group Concentration } \\
\left(\mathbf{u g} \cdot \mathbf{m L}^{-1}\right)\end{array}$} & \multicolumn{2}{c|}{$\begin{array}{c}\text { Hypoxic group Concentration } \\
\text { (ug· } \mathbf{m L}^{-1} \text { ) }\end{array}$} \\
\cline { 2 - 5 } & Original & Adjusted & Original & Adjusted \\
\hline $30-60$ & $238.75 \pm 2.22$ & $251.48 \pm 2.33$ & $170.91 \pm 5.26$ & $168.91 \pm 5.20^{*}$ \\
\hline $60-90$ & $287.28 \pm 1.38$ & $294.46 \pm 1.41$ & $120.95 \pm 3.50$ & $120.15 \pm 3.48^{*}$ \\
\hline $90-120$ & $280.49 \pm 1.29$ & $285.62 \pm 1.27$ & $218.61 \pm 4.28$ & $205.12 \pm 4.02^{*}$ \\
\hline $120-150$ & $256.23 \pm 6.24$ & $265.20 \pm 6.46$ & $109.43 \pm 4.16$ & $107.07 \pm 4.07 *$ \\
\hline Average & $265.69 \pm 22.36$ & $274.19 \pm 18.10$ & $154.97 \pm 50.11$ & $150.31 \pm 45.21 *$ \\
\hline
\end{tabular}

$* \mathrm{P}<0.05$ Compared with normoxic group. $\mathrm{C}_{0}=361.50 \mathrm{ug} \cdot \mathrm{mL}^{-1}$.

Figure 4. $\mathbf{P}_{\text {eff }}$ of levofloxacin is enhanced by hypoxia in the situ single-pass perfused rat intestinal model.

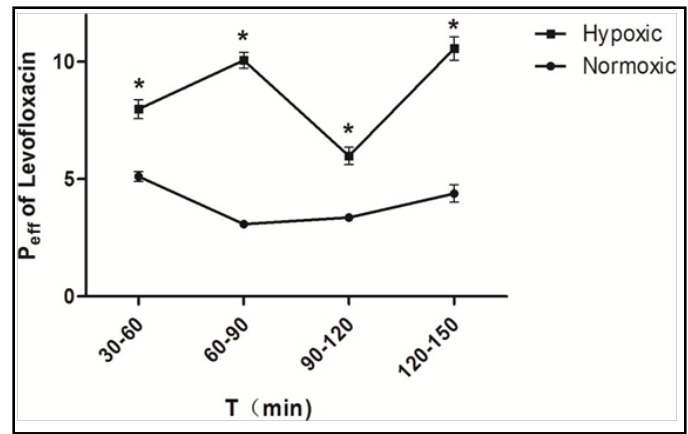

Hypoxic group rats were exposed to the high altitude for 3 days. Each data was demonstrated as mean \pm S.D. $(n=6)$.

Figure 5. Plasma concentration-time profiles of levofloxacin.

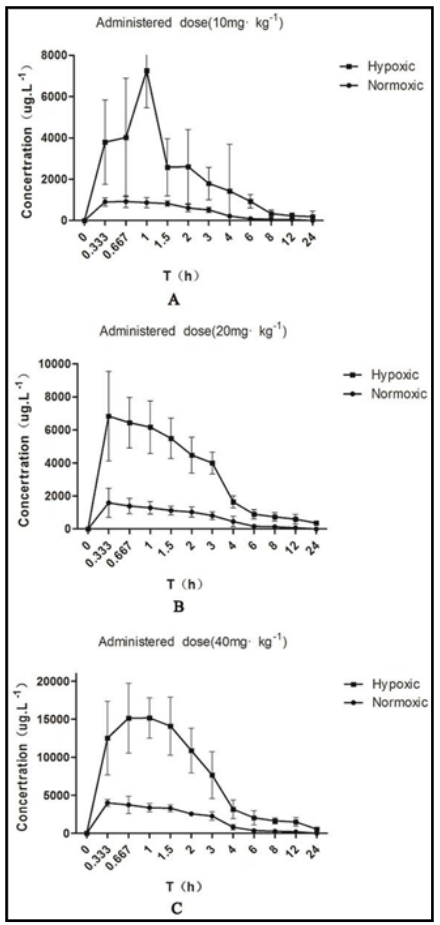

Hypoxic group rats were exposed to the high altitude for 3 days, Each group was orally administered a single dose of levofloxacin

$\left(\mathrm{A}: 10 \mathrm{mg}^{\bullet} \mathrm{kg}^{-1} ; \mathrm{B}: 20 \mathrm{mg}^{\bullet} \mathrm{kg}^{-1} ; \mathrm{C}: 40 \mathrm{mg}^{\bullet} \mathrm{kg}^{-1}\right)$. Each data was demonstrated as mean \pm S.D. $(\mathrm{n}=6)$. 
Table 3. Pharmacokinetics of levofloxacin ( $X \pm S, n=6)$.

\begin{tabular}{|c|c|c|c|c|c|c|c|}
\hline \multirow{2}{*}{ Parameters } & \multirow{2}{*}{ Units } & \multicolumn{2}{|c|}{ Administered dose $\left(10 \mathrm{mg}^{\bullet} \mathrm{kg}^{-1}\right)$} & \multicolumn{2}{|c|}{ Administered dose $\left(20 \mathrm{mg}^{\circ} \mathrm{kg}^{-1}\right)$} & \multicolumn{2}{|c|}{ Administered dose $\left(40 \mathrm{mg} \bullet \mathrm{kg}^{-1}\right)$} \\
\hline & & Normoxic group & Hypoxic group & Hypoxic group & Normoxic group & Hypoxic group & Normoxic group \\
\hline $\mathrm{AUC}(0-\mathrm{t})$ & ug/L*h & $2828 \pm 455$ & $17119 \pm 8747^{*}$ & $4803 \pm 1494$ & $28342 \pm 5715^{*}$ & $12445 \pm 1614$ & $60362 \pm 21044^{*}$ \\
\hline $\operatorname{AUC}(0-\infty)$ & ug/L*h & $2886 \pm 464$ & $18404 \pm 10312^{*}$ & $5071 \pm 1563$ & $36352 \pm 11554^{*}$ & $13307 \pm 942$ & $67818 \pm 20490^{*}$ \\
\hline $\operatorname{MRT}(0-\mathrm{t})$ & $\mathrm{h}$ & $3.01 \pm 0.53$ & $5.20 \pm 1.62 *$ & $3.01 \pm 0.35$ & $5.91 \pm 0.56^{*}$ & $3.35 \pm 0.79$ & $5.23 \pm 0.99 *$ \\
\hline $\operatorname{MRT}(0-\infty)$ & $\mathrm{h}$ & $3.95 \pm 1.36$ & $9.36 \pm 7.27 *$ & $3.97 \pm 0.67$ & $17.49 \pm 18.03^{*}$ & $5.01 \pm 2.27$ & $8.79 \pm 2.19 *$ \\
\hline $\mathrm{t} 1 / 2 \mathrm{z}$ & $\mathrm{h}$ & $2.34 \pm 0.61$ & $6.74 \pm 5.24^{*}$ & $3.11 \pm 0.42$ & $15.27 \pm 17.42^{*}$ & $5.7 \pm 3.81$ & $8.68 \pm 2.77^{*}$ \\
\hline $\operatorname{Tmax}$ & $\mathrm{h}$ & $0.45 \pm 0.20$ & $1.04 \pm 1.46^{*}$ & $0.46 \pm 0.25$ & $0.42 \pm 0.31 *$ & $0.38 \pm 0.14$ & $0.67 \pm 0.21 *$ \\
\hline $\mathrm{CLz} / \mathrm{F}$ & $\mathrm{L} / \mathrm{h} / \mathrm{kg}$ & $1.42 \pm 0.26$ & $0.31 \pm 0.21 *$ & $0.85 \pm 0.23$ & $0.12 \pm 0.04 *$ & $0.31 \pm 0.02$ & $0.07 \pm 0.03 *$ \\
\hline $\mathrm{Vz} / \mathrm{F}$ & $\mathrm{L} / \mathrm{kg}$ & $4.79 \pm 1.40$ & $2.61 \pm 2.01 *$ & $3.77 \pm 1.07$ & $2.1 \pm 1.69^{*}$ & $2.49 \pm 1.71$ & $0.83 \pm 0.4^{*}$ \\
\hline $\mathrm{Cmax}$ & $\mathrm{ug} / \mathrm{L}$ & $977 \pm 285$ & $6874 \pm 6208^{*}$ & $1655 \pm 834$ & $7078 \pm 2454^{*}$ & $4175 \pm 702$ & $16175 \pm 3391 *$ \\
\hline
\end{tabular}

$* \mathrm{P}<0.05$, Compared with normoxic group.

Additionally, higher efficiency of drug efflux and increased and stable absorption was observed over time when the oxygen supply was sufficient. On the contrary, the slope of the absorption curve was steeper when oxygen was inadequate, indicating that hypoxia significantly inhibited P-gp mediated levofloxacin efflux. The everted intestinal sac model had the intestine immersed in simulated body fluid with no blood supply to the segments, which eliminated this potential confounding factor. Subsequently in the model of the in-situ single-pass perfused rat intestine, the Peff of levofloxacin was significantly higher in the hypoxic group compared to that in the normoxic group, indicating that levofloxacin penetrated the membrane of the intestinal wall more easily under hypoxia. As a specific substrate of P-gp, the increased Peff of levofloxacin indicated decreased efflux by P-gp, which was consistent with the downregulated P-gp expression and supported the aforementioned hypothesis. The in-situ single-pass perfused intestinal model maintains the drug solution under the laminar flow condition, which is important for determining the true membrane permeability of drugs. However, it is an invasive approach; the rats were anesthetized during the perfusion process, which possibly affected their heart rate and cardiac output while causing other physiological symptoms that could have impacted drug absorption. To eliminate this influence, the rats were orally administered levofloxacin in the third model to directly detect drug absorption. The results showed that the pharmacokinetic parameters of levofloxacin at low, medium, and high doses were significantly changed under hypoxia. The AUC and Cmax of levofloxacin, representing the degree of absorption, substantially increased after hypoxia, indicating that an increased amount of levofloxacin entered the body. These results were consistent with the findings from the other two models. Therefore, all three models support the hypothesis.

On one hand, an increased drug concentration can improve its therapeutic effect, but on the other hand, it may cause serious adverse reactions. Gastrointestinal symptoms such as abdominal discomfort or pain, diarrhea, nausea, or vomiting are the main adverse effects of levofloxacin. While these may be aggravated, other adverse events such as seizures, mental disorders, irritability, confusion, hallucinations, and tremors may often occur at high altitudes. To ensure drug safety at high altitudes, it may be necessary to adjust the usage and dosage of levofloxacin to maintain a safe concentration within the therapeutic window. This applies not only to levofloxacin but to other substrates of P-gp as well, particularly those with narrow therapeutic windows. Thus, therapeu- tic drug monitoring (TDM) in imperative; for example, special attention should be paid to the occurrence of adverse reactions to digoxin when used at high altitudes $[2,5]$. However, some serious adverse reactions may occur before the plasma concentration changes, and TDM may not be able to predict adverse reactions in a timely manner. For instance, loperamide is a powerful antidiarrheal drug and also a substrate of P-gp; few nervous system related adverse reactions have been reported in response to this drug because the level of loperamide that could enter the central nervous system is low when it is used alone, and most of the drug is removed from the nervous system by P-gp. Nevertheless, when used in combination with quinidine, which is an inhibitor of P-gp and decreases its efflux activity, more loperamide can enter the nervous system and bind to opioid receptors, resulting in respiratory depression. A similar situation may arise under hypoxia when P-gp expression is downregulated. Importantly, respiratory depression may emerge before an increased concentration of loperamide can be detected in the blood. Previous data have shown that the concentration of loperamide increased $60 \mathrm{~min}$ after coadministration with quinidine, while the adverse event occurred at $30 \mathrm{~min}[13]$, by which time it was too late to adjust the dosage. Therefore, to monitor adverse drug reactions, understanding the changes in drug transporters under hypoxia is as important as performing TDM. In conclusion, hypoxia can downregulate P-gp expression and increase the absorption of levofloxacin in the intestine at high altitudes, thereby enhancing its plasma concentration. Thus, understanding the variations and the mechanisms of action of drug transporters under hypoxia is important for informed drug administration at high altitudes.

\section{Acknowledgements}

We are grateful for department of pharmacy of the second military medical university, thanks for their support and housing at the investigation site to facilitate this study. Special thanks to Dr. Cao Yan for the technical assistance in our research.

\section{References}

[1]. Bush JA, Li G. Regulation of the Mdr1 isoforms in a p53-deficient mouse model. Carcinogenesis. 2002 Oct;23(10):1603-7. Pubmed PMID: 12376467.

[2]. Charoo NA, Abdallah DB, Parveen T, Abrahamsson B, Cristofoletti R, Groot DW, et al. Biowaiver Monograph for Immediate-Release Solid Oral Dosage Forms: Moxifloxacin Hydrochloride. J Pharm Sci. 2020 Sep;109(9):26542675. Pubmed PMID: 32534881. 
[3]. Ding Z, Yang L, Xie X, Xie F, Pan F, Li J, et al. Expression and significance of hypoxia-inducible factor-1 alpha and MDR1/P-glycoprotein in human colon carcinoma tissue and cells. J Cancer Res Clin Oncol. 2010 Nov;136(11):1697-707. Pubmed PMID: 20217131.

[4]. Dopp JM, Moran JJ, Abel NJ, Wiegert NA, Cowgill JB, Olson EB, et al. Influence of intermittent hypoxia on myocardial and hepatic P-glycoprotein expression in a rodent model. Pharmacotherapy. 2009 Apr;29(4):365-72. Pubmed PMID: 19323616.

[5]. Drescher S, Glaeser H, Mürdter T, Hitzl M, Eichelbaum M, Fromm MF. P-glycoprotein-mediated intestinal and biliary digoxin transport in humans. Clin Pharmacol Ther. 2003 Mar;73(3):223-31. Pubmed PMID: 12621387.

[6]. Fradette C, Du Souich P. Effect of hypoxia on cytochrome P450 activity and expression. Curr Drug Metab. 2004 Jun;5(3):257-71. Pubmed PMID: 15180495.

[7]. Fradette C, Batonga J, Teng S, Piquette-Miller M, du Souich P. Animal models of acute moderate hypoxia are associated with a down-regulation of CYP1A1, 1A2, 2B4, 2C5, and 2C16 and up-regulation of CYP3A6 and Pglycoprotein in liver. Drug Metab Dispos. 2007 May;35(5):765-71. Pubmed PMID: 17303624

[8]. Ho GT, Moodie FM, Satsangi J. Multidrug resistance 1 gene (P-glycoprotein 170): an important determinant in gastrointestinal disease? Gut. 2003 May;52(5):759-66. Pubmed PMID: 12692067.

[9]. Hunter FW, Young RJ, Shalev Z, Vellanki RN, Wang J, Gu Y, et al. Identification of P450 Oxidoreductase as a Major Determinant of Sensitivity to Hypoxia-Activated Prodrugs. Cancer Res. 2015 Oct 1;75(19):4211-23. Pubmed PMID: 26297733.

[10]. Jürgens G, Christensen HR, Brøsen K, Sonne J, Loft S, Olsen NV. Acute hypoxia and cytochrome P450-mediated hepatic drug metabolism in humans. Clin Pharmacol Ther. 2002 Apr;71(4):214-20. Pubmed PMID: 11956503.

[11]. Maillefert JF, Maynadie M, Tebib JG, Aho S, Walker P, Chatard C, et al. Expression of the multidrug resistance glycoprotein 170 in the peripheral blood lymphocytes of rheumatoid arthritis patients. The percentage of lymphocytes expressing glycoprotein 170 is increased in patients treated with prednisolone. Br J Rheumatol. 1996 May;35(5):430-5. Pubmed PMID: 8646432.

[12]. Rahman MS, Thomas P. Effects of hypoxia exposure on hepatic cytochrome P450 1A (CYP1A) expression in Atlantic croaker: molecular mechanisms of CYP1A down-regulation. PLoS One. 2012;7(7):e40825. Pubmed PMID: 22815834.

[13]. Sadeque AJ, Wandel C, He H, Shah S, Wood AJ. Increased drug delivery to the brain by P-glycoprotein inhibition. Clin Pharmacol Ther. 2000 Sep;68(3):231-7. Pubmed PMID: 11014404.

[14]. Sharom FJ. The P-glycoprotein multidrug transporter. Essays in biochemistry. 2011 Sep 7;50:161-78.

[15]. Verstraelen J, Reichl S. Upregulation of P-glycoprotein expression by ophthalmic drugs in different corneal in-vitro models. J Pharm Pharmacol. 2015 May;67(5):605-15. Pubmed PMID: 25643948.

[16]. Wartenberg M, Ling FC, Müschen M, Klein F, Acker H, Gassmann M, et al. Regulation of the multidrug resistance transporter P-glycoprotein in multicellular tumor spheroids by hypoxia-inducible factor (HIF-1) and reactive oxygen species. FASEB J. 2003 Mar;17(3):503-5. Pubmed PMID: 12514119.

[17]. Yamada T, Takaoka AS, Naishiro Y, Hayashi R, Maruyama K, Maesawa C, et al. Transactivation of the multidrug resistance 1 gene by T-cell factor 4/ beta-catenin complex in early colorectal carcinogenesis. Cancer Res. 2000 Sep 1;60(17):4761-6. Pubmed PMID: 10987283.

[18]. Yu H, Shao H, Wu Q, Sun X, Li L, Li K, et al. Altered gene expression of hepatic cytochrome $\mathrm{P} 450$ in a rat model of intermittent hypoxia with emphysema. Mol Med Rep. 2017 Jul;16(1):881-886. Pubmed PMID: 28560400.

[19]. Zhou SF. Structure, function and regulation of P-glycoprotein and its clinical relevance in drug disposition. Xenobiotica. 2008 Jul;38(7-8):802-32. Pubmed PMID: 18668431. 